\title{
A NOVEL LATTICE BOLTZMANN METHOD FORMULATION FOR AN AXISYMMETRIC FLOW WITH SWIRL IN VORTICITY-STREAM FUNCTION VARIABLES.
}

\author{
S. Pedraza ${ }^{1}$, O. D. López ${ }^{2}$, J. R. Toro ${ }^{3}$ \\ ${ }^{1}$ Graduate Research Assitant, Computational Mechanics Research Group, Department of Me- \\ chanical Engineering, Universidad de los Andes, Bogotá, Colombia. \\ (se-pedra@uniandes.edu.co) \\ 2 Assistant Professor, Computational Mechanics Research Group, Universidad de los Andes, \\ Bogotá, Colombia.
}

${ }^{3}$ Professor, Computational Mechanics Research Group, Universidad de los Andes, Bogotá, Colombia.

\begin{abstract}
A novel lattice Boltzmann formulation for axisymmetric flows with swirl is proposed in vorticity-stream function variables. The present model solves the evolution equations for the azimuthal components of the velocity and vorticity coupled with the Poisson equation for the stream function. All three equations are solved by using source terms in a pseudo cartesian lattice Boltzmann formulation (LBM) formulation, which are needed in order to modify the standard D2Q5 lattice model used in the study. Numerical results of the simulations of a lid-driven swirling flow in a confined cylindrical cavity shows good agreement between the proposed model and numerical and experimental data available in the literature.
\end{abstract}

Keywords: Vorticity-stream function formulations, LBM, sources, axisymmetric flow with swirl.

\section{INTRODUCTION}

The Lattice Boltzmann Method (LBM) has shown to be an efficient solver for the Navier-Stokes (NS) equations and for some other non-linear partial differential equations [6],[9]. This so called efficiency, apart of its programming simplicity, is based on the fact that any term that is not achieved through the multiscale analysis can be introduced in the LBM formulation as a source term. Several authors have studied the insertion of this sources willing to achieve forcing terms in evolution equations, sources in Poisson's equations or to complete operators that owing, for example, the discrepancy between the dimension of the lattice and the dimension of the flow do not appear through the multiscale analysis.

Swirling flows can be observed in different phenomena ranging from nature to engineering applications. For example in combustion systems such as turbines, burners or boilers swirling flows are artificial induced in order to improve the mixing rate between the fuel and oxidant [3]. In aeronautics, delta winged aircraft produce leading-edge vortex that benefit the lift and stability but sometimes under certain conditions this vortex can undergo structural changes such as vortex breakdowns (O Lucca, Peckam and Atkinson 1957). In 1960 Harvey 
[1] recognized the appearance of this structural changes within swirling flows in cylindrical tubes and by 1980 Escudier [1] using a cylindrical container with a rotating endwall and laser-induced fuorescein dye fully characterized the occurrence of this vortex breakdowns as function of Reynolds $(\mathrm{Re})$ number and container aspect ratio.

A vortex breakdown refers to a disturbance characterized by the formation of an internal stagnation point on the vortex axis, followed by a reverse flow [2]. Lugt and Haussling [1] were the first to run numerical simulations restricting to small aspect ratios or small $\mathrm{Re}$ numbers where no vortex breakdown took place. By 1987 Lugt and Abboud simulate accurately the vortex breakdown phenomena using as a benchmark the available experimentally results by Escudier. In 1990, using a finite difference scheme, J.M Lopez studied axisymmetric vortex breakdown phenomena in a confined swirling flow in order to explore the physical mechanisms behind it. Up to date, Bhaumik 2007 [4], using a LBM 3D scheme simulated a lid-driven flow within a confined cylindrical cavity. In 2008, a hybrid LBM axysimmetric formulation was proposed by Huang [7] solving the underlying 2D flow with a pressure-velocity formulation coupled with a finite difference scheme for the azimuthal velocity for the TaylorCouette flow. It is worth to be mentioned that the present study seeks to solve the flow using only one methodology instead of formulating a different kind of lattice. The simulations were carried out using a multiple relaxation method in order to solve the Navier-Stokes equations in pressure velocity variables but somehow ignoring the axisymmetrical characteristic of the flow. In the present study, a CFD simulation of a lid-driven cylindrical confined flow with aspect ratio 2.5 was used as a benchmark for the LBM simulations. The proposed LBM formulation results showed good agreement with the CFD results for $\operatorname{Re}<1000$ but tend to diverge when the Re number was increased. The authors have not found the issue behind this so called divergence but are still working in order to improve the LBM formulation that is proposed in the present study.

\section{GOVERNING EQUATIONS}

The flow problem considered in the present study conisist in cylindrical container with radius $R$, height $H$ which is lid-driven on the top (or bottom) by a constant angular velocity $\Omega$.

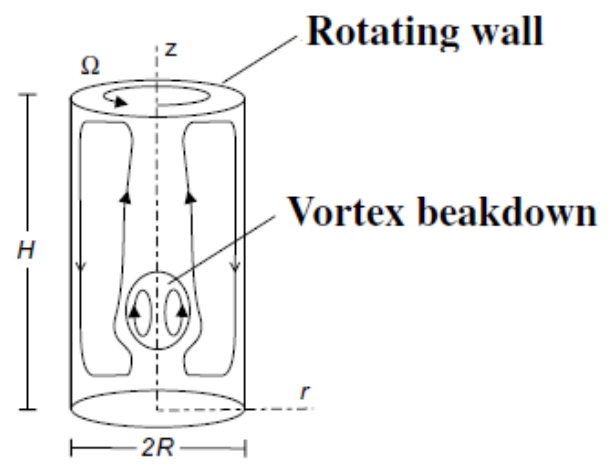

Figure 1. Lid-driver cavity. Taken from []

The fluid that fills the cylindrical container is incompressible with constant cinematic 
viscosity $\nu$. The axysimmetric formulation for the Navier-Stokes equations is employed considering the velocity field $\left(u_{r}, v, u_{z}\right)$ in cylindrical coordinates $(r, \theta, z)$. The Navier-Stokes equations are solved using the vorticity-stream function formulation where

$$
u_{r}=-\frac{1}{r} \frac{\partial \psi}{\partial z}, u_{z}=\frac{1}{r} \frac{\partial \psi}{\partial r}
$$

which satisfies continuity $\left(\nabla \cdot \vec{u}=-u_{r} v / r\right)$ and is used in Poissons equation to recover the vorticity field

$$
\omega=-\frac{1}{r} \frac{\partial^{2} \psi}{\partial^{2} z}-\frac{\partial}{\partial r}\left(\frac{1}{r} \frac{\partial \psi}{\partial r}\right)
$$

Introducing (1) and (2) into the Navier-Stokes equations leads to the following evolution equations for the azimuthal veloctiy and azimuthal vorticity

$$
\begin{gathered}
\frac{\partial v}{\partial t}+u_{r} \frac{\partial v}{\partial r}+u_{z} \frac{\partial v}{\partial z}=-u_{r} \frac{v}{r}+\frac{1}{R e}\left[\frac{\partial^{2} v}{\partial z^{2}}+\frac{\partial^{2} v}{\partial r^{2}}+\frac{\partial v}{r \partial r}-\frac{v}{r^{2}}\right] \\
\frac{\partial \omega}{\partial t}+u_{r} \frac{\partial \omega}{\partial r}+u_{z} \frac{\partial \omega}{\partial z}=u_{r} \frac{\omega}{r}+2 \frac{v}{r} \frac{\partial v}{\partial z}+\frac{1}{R e}\left[\frac{\partial^{2} \omega}{\partial z^{2}}+\frac{\partial^{2} \omega}{\partial r^{2}}+\frac{\partial \omega}{r \partial r}-\frac{\omega}{r^{2}}\right]
\end{gathered}
$$

together with the Poisson equation,

$$
\frac{\partial^{2} \psi}{\partial z^{2}}+\frac{\partial^{2} \psi}{\partial r^{2}}-\frac{\partial \psi}{r \partial r}=-r \omega
$$

the continuity equation $\nabla \cdot \vec{u}=-u_{r} v / r$ and the boundary conditions dictated by

$$
\begin{aligned}
& \psi(0, z)=v(0, z)=\omega(0, z)=0 \\
& \psi(1, z)=v(1, z)=0, \omega(1, z)=-\frac{1}{r} \frac{\partial^{2} \psi}{\partial r^{2}} \\
& \psi(r, 0)=0, \quad v(r, 0)=\Omega r, \omega(r, 0)=-\frac{1}{r} \frac{\partial^{2} \psi}{\partial z^{2}} \\
& \psi(r, H)=v(r, H)=0, \omega(r, H)=-\frac{1}{r} \frac{\partial^{2} \psi}{\partial z^{2}}
\end{aligned}
$$

\section{LBM FORMULATION}

In order to develop a LBM formulation a D2Q5 lattice was chosen. The D2Q5 lattice is a two dimensional lattice with 5 posible directions of particle propagation and is widely used for advection-diffusion problems (Shen 2012). The characteristics of the lattice are: $c_{s}^{2}=2 / 5, c_{0}=(0,0), c_{1}=(1,0), c_{2}=(0,1), c_{3}=(-1,0), c_{4}=(0,-1)$ and the lattice weight parameters are $t_{i}=1 / 5$. In the present study, only the azimuthal velocity formulation is described due to the similarities of the evolution equations (3) and (4).

First consider the following equilibrium distribution

$$
f_{i}^{e q}=\frac{v}{5}\left[1+2.5 u \cdot c_{i}\right]
$$


where

$$
\sum_{i} c_{i} f_{i}^{e q}=v u, \quad \sum_{i} c_{i} c_{i} f_{i}^{e q}=c_{s}^{2} v \text { and } u=\left(u_{r}, u_{z}\right) .
$$

The evolution equation, for the LBM formulation, and its second order taylor approximation are described as

$$
\begin{aligned}
f_{i}\left(x+c e_{i}, t+1\right)-f_{i}(x, t) & =-\frac{1}{\tau}\left[f_{i}-f_{i}^{e q}\right]+h_{i} \\
D_{i} f_{i}+\frac{1}{2} D_{i}^{2} f_{i} & =-\frac{1}{\tau}\left[f_{i}-f_{i}^{e q}\right]+h_{i}
\end{aligned}
$$

where $f_{i}=f_{i}^{(0)}+\epsilon f_{i}^{(1)}+\epsilon f_{i}^{(2)}, D_{i}=\partial_{t}+\nabla \cdot c_{i}, \partial_{t}=\epsilon \partial_{t 1}+\epsilon^{2} \partial_{t 2}, h_{i}=\epsilon h_{i}^{(1)}+\epsilon^{2} h_{i}^{(2)}$ (source terms) and $\nabla=\epsilon \nabla$. Then, using the multiscale expansion of each term, terms of order $O(1)$

$$
f_{i}^{(0)}=f_{i}^{e q}
$$

$\operatorname{order} O(\epsilon)$

$$
\partial_{t 1} f_{i}^{(0)}+\left(\nabla \cdot c_{i}\right) f_{i}^{(0)}=-\frac{1}{\tau} f_{i}^{(1)}+h_{i}^{(1)}
$$

and order $O\left(\epsilon^{2}\right)$

$$
\partial_{t 2} f_{i}^{(0)}+\frac{1}{2} \partial_{t 1}^{2} v+\partial_{t 1}\left(\nabla \cdot c_{i}\right) f_{i}^{(0)}+\frac{1}{2}\left(\nabla \nabla: c_{i} c_{i}\right) f_{i}^{(0)}+\partial_{t 1} f_{i}^{(1)}+\left(\nabla \cdot c_{i}\right) f_{i}^{(1)}=\frac{1}{\tau} f_{i}^{(2)}+h_{i}^{(2)} .
$$

are considered.

Taking in account that probability function distributions are being handled, the next step is to calculate zeroth and first order moments for the distributions. Due to the discrete space, a summation is taken over the directions of the lattice to the latter equations in order to achieve evolution equations for the macroscopic variables. (Eqs. 3, 4 and 5)

Taking the zeroth moment of the equation (15) and considering that $\sum f_{i}^{(1)}=0$ the $e_{\theta}$ component of the Euler equation is found to be

$$
\begin{aligned}
\partial_{t 1} v+\nabla \cdot(v u) & =H^{(1)} \\
\partial_{t 1} v+v(\nabla \cdot u)+u \cdot \nabla v & =H^{(1)}
\end{aligned}
$$

where $H^{(j)}=\sum h_{i}^{(j)}$.

Taking the first moment of the probability function on the (15) equation and introducing it into the zeroth moment of the equation (16) was obtained

$\tau \nabla \cdot\left(\sum c_{i} h_{i}^{(1)}-\partial_{t 1}(v u)-c_{s}^{2} \nabla v\right)+\frac{1}{2}\left(2 \partial_{t 2} v+\partial_{t 1} H^{(1)}-\partial_{t 1} \nabla \cdot(v u)+2 \partial_{t 1} \nabla \cdot(v u)+c_{s}^{2} \nabla^{2} v\right)=H^{(2)}$

After some manipulations and considering that $h_{i}=\epsilon h_{i}^{(1)}+\epsilon^{2}\left(h_{i}^{(2)}+\partial_{t 1} h_{i}^{(1)} / 2\right)$ the equation (19) reads

$$
\partial_{t} v+v(\nabla \cdot u)+u \cdot \nabla v+\tau \nabla \cdot \sum c_{i} h_{i}^{(1)}+\left(\frac{1}{2}-\tau\right)\left(\partial_{t 1} \nabla \cdot(v u)+c_{s}^{2} \nabla^{2} v\right)=H^{(1)}+H^{(2)}
$$


defining $\nu_{l b m}=(\tau-1 / 2) c_{s}^{2}$ in order to achieve an advection-diffusion equation.

As mentioned in the introduction of the present study the reason for introducing source terms is the discrepancy between the lattice dimension and the dimensional nature of the flow. The lid-driven cavity flow is said to be a "two-and-a-half dimensional flow" due to the flow in the $e_{\theta}$ is predetermined by the underlying 2D flow, i.e. $v$ does not depend on the $\theta$ coordinate. Considering that a D2Q5 lattice model is used, "half dimension" is not captured through the lattice causing that the operators achieved in the multiscale analysis are not the same as the operators that naturally arise in the flow description i.e. the operators through LBM are "cartesian" operators while the operators in the evolution equations are "cylindrical". For example, consider a vector field $A$ and its divergence in cylindrical coordinates and in cartesian coordinates

$$
\nabla_{c i l} \cdot A=\frac{1}{r} \frac{\partial}{\partial r}\left(r A_{r}\right)+\frac{\partial A_{z}}{\partial z} \neq \frac{\partial A_{r}}{\partial r}+\frac{\partial A_{z}}{\partial z}=\nabla_{\text {cart }} \cdot A .
$$

This also happens for the $\Delta=\nabla \cdot \nabla$ operator and the discrepancy in both operators is manifested in equations (18) and (20) forcing the introduction of source terms in the formulation.

\subsection{Restrictions on the source term}

The equation (18) is intended to reproduce the Euler equation in the $e_{\theta}$ coordinate forcing the definition of $H^{(1)}$ to be

$$
H^{(1)}=-u_{r} \frac{v}{r}
$$

The second restriction on the source term of first order arises from the equation (20) where the term $\tau \nabla \cdot \sum c_{i} h_{i}^{(1)}$ appears. Observing the azimuthal evolution equation (3) the latter term does not appear, forcing it to be zero. Considering both restrictions the source term of first order is completely defined as

$$
h_{i}^{(1)}=-\xi_{i} u_{r} \frac{v}{r}
$$

where $\xi_{0}=0$ and $\xi_{i>0}=1 / 4$.

The manipulation for the source term of second order, $H^{2}$, is the same as the described above leading to

$$
h_{i}^{(2)}=\xi_{i} \nu_{l b m}\left[\frac{\partial v}{r \partial r}-\frac{v}{r^{2}}\right]
$$

where the space derivative of $v$ is calculated through a finite difference scheme using the lattice grid.

The source terms for the azimuthal vorticity are calculated in the same way as the preceding terms considering only that the inviscid evolution equation for the azimuthal equation is

$$
\partial_{t} \omega+u \nabla \cdot \omega=\omega \frac{u_{r}}{r}+2 \frac{v}{r} \frac{\partial v}{\partial z}
$$


leading the first order source term to be

$$
h_{i}^{(1)}=\xi_{i}\left(\omega \frac{u_{r}}{r}+2 \frac{v}{r} \frac{\partial v}{\partial z}\right)
$$

and

$$
h_{i}^{(2)}=\xi_{i} \nu_{l b m}\left[\frac{\partial \omega}{r \partial r}-\frac{\omega}{r^{2}}\right]
$$

\subsection{Source terms for the Poisson equation}

Considering the model proposed by (Chai), where the equilibrium distribution is defined as

$$
f_{e q}= \begin{cases}\left(t_{0}-1\right) \psi & i=0 \\ t_{i} \psi & i>0\end{cases}
$$

with $\sum_{i=0}^{4} t_{i}=1, t_{0}=0$ and

$$
\psi(x, t)=\sum_{i=1}^{4} f_{i}^{e q}(x, t)
$$

the following source term of second order is introduced into the formulation

$$
h_{i}^{(2)}=\xi_{i} D\left(-r \omega+u_{z}\right)
$$

where $D=\alpha(1 / 2-\tau)$ and $\alpha$, a constant which depends on the lattice model being for a D2Q5 model $\alpha=1 / 2$.

\section{NUMERICAL RESULTS}

First, the introduction of source terms in the Poisson LBM formulation was tested due to the fact that the parameters proposed by Chai [6] are somehow arbitrarily chosen, specifically $\tau$ which is set to 1 . Consider so the following stream function

$$
\psi(x, y)=x^{3} \sin (\pi x) \sin (\pi y), \quad(x, y) \in D=[0,1] \times[0,1]
$$

which is null in the frontier of $\mathrm{D}$. Introducing $\psi(x, y)$ into the Poisson equation (15) the following vorticity scalar field is obtained

$$
\begin{aligned}
\omega(x, y)= & -\sin (\pi y)\left(\left(6-\pi^{2} x^{2}\right) \sin (\pi x)+6 \pi x \cos (\pi x)\right) \\
& +\pi^{2} x^{3} \sin (\pi x) \sin (\pi y)+\sin (\pi y)(3 \sin (\pi x)+\pi x \cos (\pi x)) .
\end{aligned}
$$

With the vorticity field and considering the boundary conditions expressed in equations $(6,7,8,9)$ the LBM alogrithm is "fed" in order to calculate the solution of the equation (15) shown in Fig.1(b). This boundary conditions, $\partial^{2} \psi / \partial x^{2}$, are calculated through a second order Taylor expansion in the boundary of the domain towards the interior.

Having tested the model proposed by Chai [6] the simulations of the lid-driven cylinder cavity were carried out. The results of the LBM formulation were examined for agreement with a CFD simulation that was run in Ansys v.14 FLUENT as a full 3D laminar flow with 


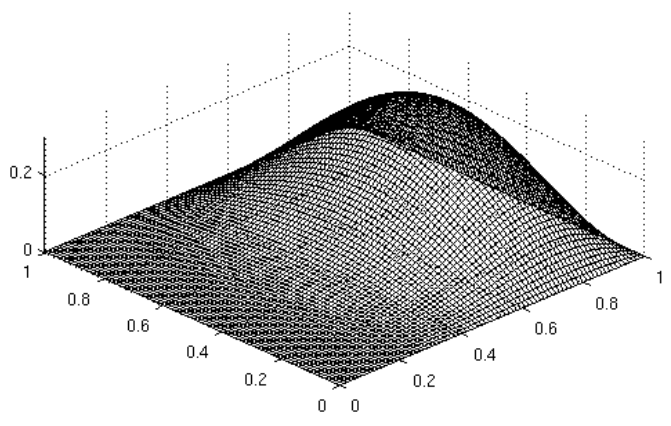

(a) Analytical solution of the equation (15) using the vorticity field of equation (32)

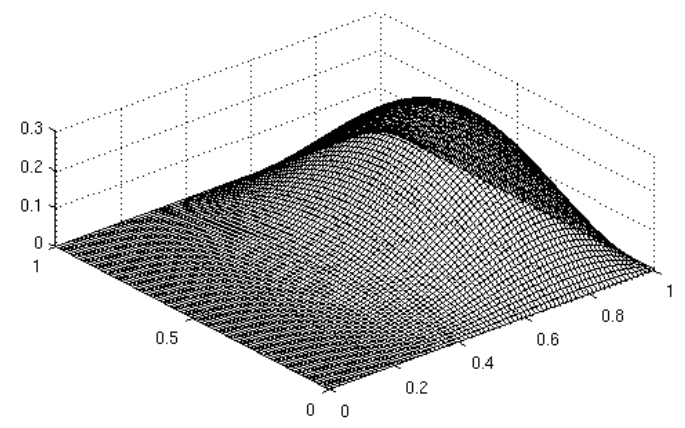

(b) Numerical solution of the equation (15) using the vorticity field of equation (32)

Figure 2. Analytical and numerical solution of the equation (15) using the vorticity field of equation (32) and boundary conditions expressed in equations $(6,7,8,9)$

Table 1. Comparisons between analytical solutions and numerical solutions

\begin{tabular}{cc}
\hline Grid spacing $\Delta x$ & $\left\|u_{\text {analytic }}(x, y)-u_{l b m}(x, y)\right\|_{2}$ \\
\hline 0.05263 & 0.0701 \\
0.02564 & 0.0356 \\
0.01694 & 0.0239 \\
0.01265 & 0.0181 \\
0.01010 & 0.0147 \\
\hline
\end{tabular}

$6 \times 10^{6}$ elements in steady state. The LBM simulations were carried out for a cylinder cavity of 2.5 aspect ratio at $\operatorname{Re} 100,300$ and 500 on a $50 \times 125$ lattice grid (Fig 3, 4 and 5). The LBM simulations iterated until the energy of the system $\left(1 / 2|| u \|^{2}\right)$ have reached a constant value while the CFD were carried out until the residuals of the velocity were less or equal than $10^{-5}$. The LBM code consumed for the Re 500 flow a c.p.u time of about $1.5 \mathrm{~h}$ while the CFD simulation took about $6 \mathrm{~h}$ of c.p.u time both run in the same computer.

\section{CONCLUSIONS}

In this paper, a new LBM formulation was proposed for the numerical solution of the vorticity-stream function evolution equations in an axysimmetric flow with swirl. The good performance of the present model is demonstrated by the agreement with FLUENT CFD simulations (Fig 3, 4 and 5). In comparison with ohter LBM formulations for axysimmetric flows the present achieves to include all the variables in the same formulation avoiding to solve hybrid schemes. Although, the formulation is not capable already to solve higher Re flows, the simulations for lower Re showed good agreement validating the source terms inclusion for the dimensional discrepancy. 


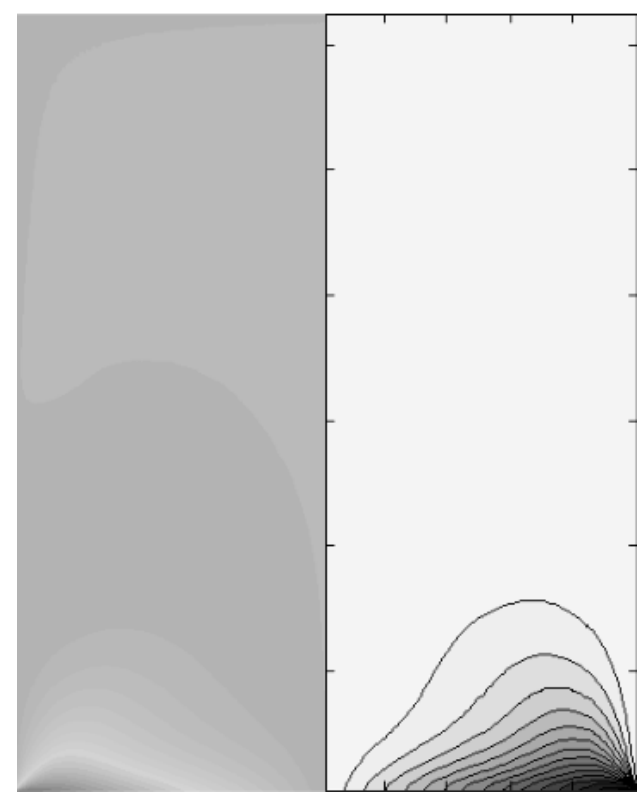

(a) CFD Solution (left) and LBM solution (Right)

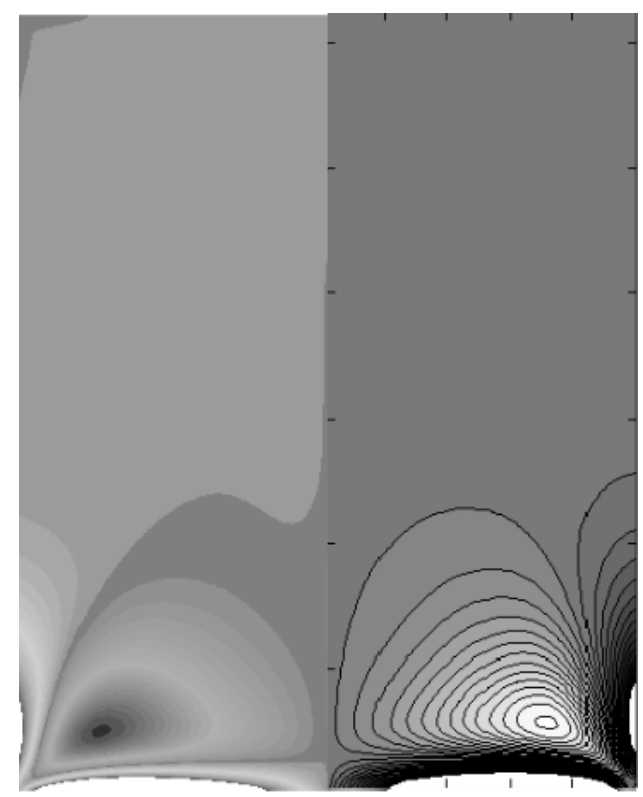

(b) CFD Solution (left) and LBM solution (Right

Figure 3. Comparison between CFD and LBM solutions of the azimuthal velocity (a) and azimuthal vorticity (b) at $\operatorname{Re} 100$

\section{REFERENCES}

[1] J.M. Lopez, "Axisymmetric vortex breakdown Part1. Confined swirlinf flow". J. Fluid Mech. 221, 533-552, 1990.

[2] S. Leibovich, " The structure of vortex breakdown". Ann. Rev. Fluid Mech. 10, 221-46, 1978.

[3] O. Lucca-Negro, T.O'Doherty, " Vortex breakdown: a review". Progress in energy combustion science. 27, 431-481, 2001.

[4] S.K. Bhaumik, K.N. Lakshmisha " Lattice Boltzmann simulation of lid driven swirling flow in confined cylindrical cavity". Computers and fluids 36, 1163-1173, 2007.

[5] L. Valino, J. martin, " Dynamics of LBM generated isotropic homogeneous turbulence with linear forcing". Third european combustion meeting ECM 2007.

[6] Zhenhua Chai " A novel Lattice Boltzmann model for the Poisson equation". Applied mathematical modelling 32, 2050-2058, 2008.

[7] H. Huang, T.S. Lee "Hybrid lattice Boltzmann finite-difference simulation of axisymmetric swirling and rotating flows". Int. J. Numer. Meth. Fluids 2007; 53:17071726.

[8] J. Latt "Hydrodynamic limit of lattice Boltzmann equations". Doctoral Thesis, Univ. Geneve; 53:17071726.

[9] Z, Chai. B.Shi, " A unified lattice Boltzmann model for some nonlinear partial differential equations". Chaos, solitons and fractals, 2008 


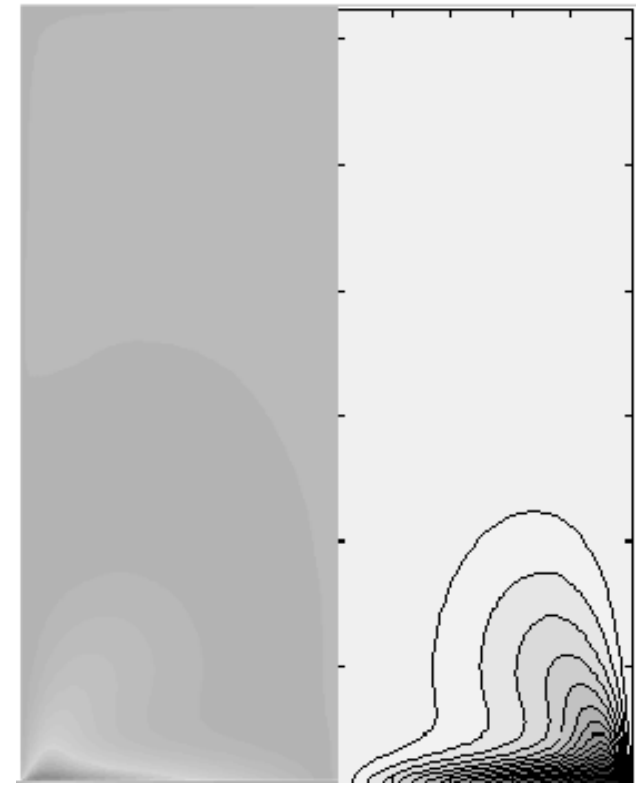

(a) CFD Solution (left) and LBM solution (Right)

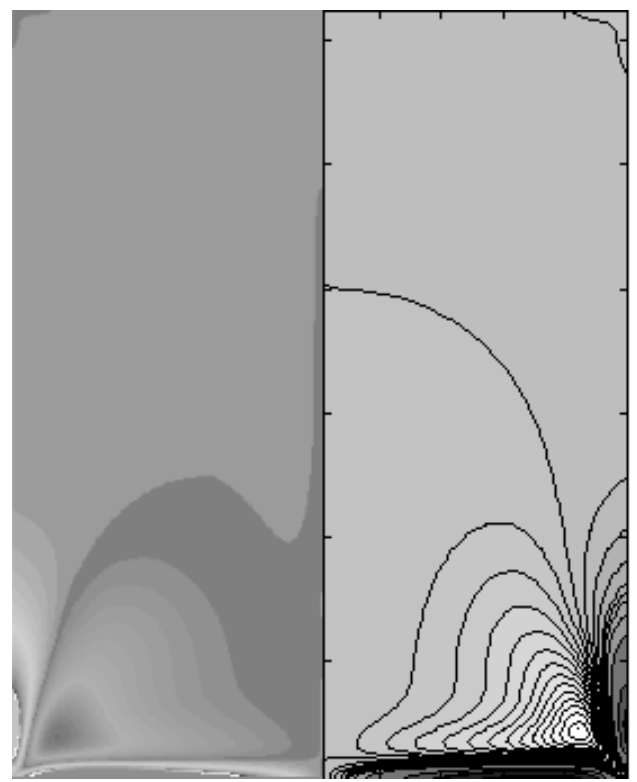

(b) CFD Solution (left) and LBM solution (Right

Figure 4. Comparison between CFD and LBM solutions of the azimuthal velocity (a) and azimuthal vorticity (b) at $\operatorname{Re} 300$

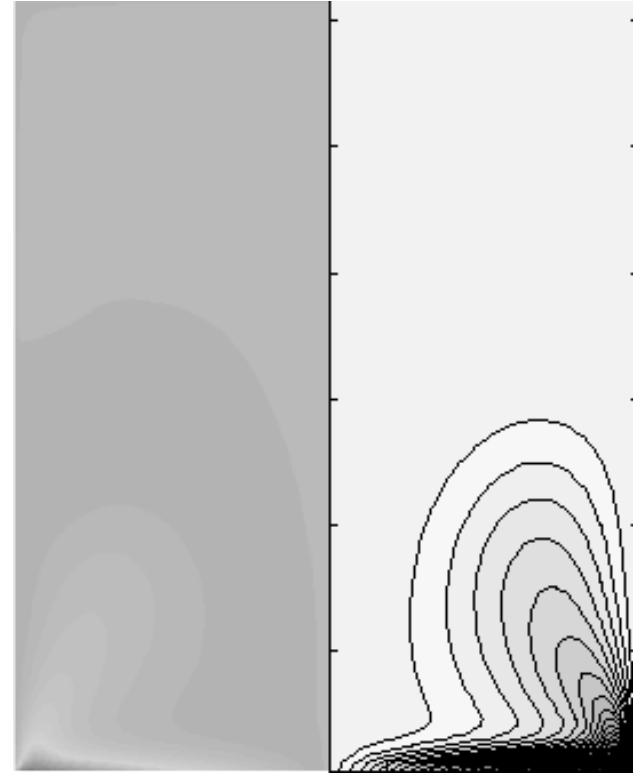

(a) CFD Solution (left) and LBM solution (Right)

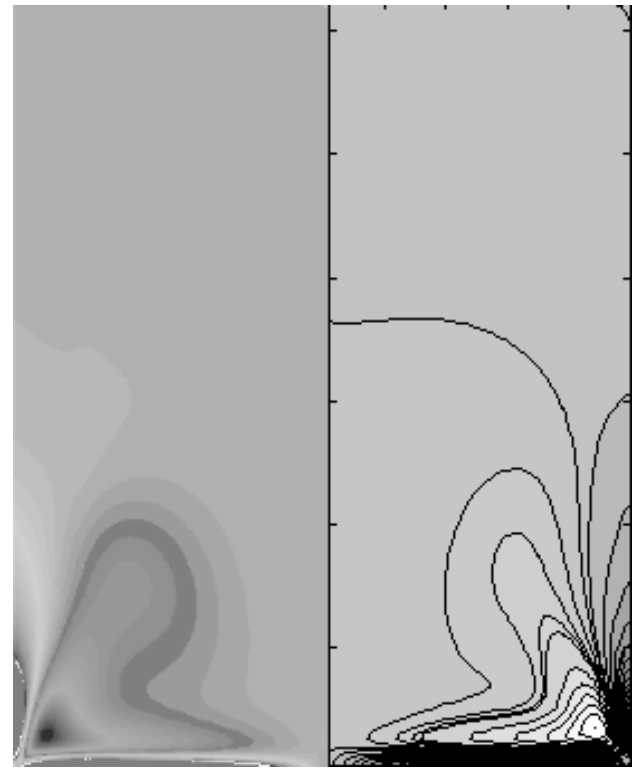

(b) CFD Solution (left) and LBM solution (Right

Figure 5. Comparison between CFD and LBM solutions of the azimuthal velocity (a) and azimuthal vorticity (b) at Re 500 